\title{
Silicon-based photonic architectures from hierarchically porous carbon opals
}

\author{
Luz Karime Gil-Herrera, ${ }^{a}$ Francisco Gallego-Gómez, ${ }^{a}$ Almudena Torres-Pardo, ${ }^{b, c}$ Jose M. González-Calbet, ${ }^{\text {b,c }}$ Francisco J. \\ Palomares, ${ }^{a}$ Alvaro Blanco, ${ }^{a}$ Beatriz H Juárez, ${ }^{d, e}$ Cefe López ${ }^{a^{*}}$
}

\begin{abstract}
Silicon-based materials are required in cutting-edge technological fields, for which hierarchical porosity and photonic properties help enhance performance. In this work, we demonstrate the versatility of several fabrication routes combining silicon infiltration by chemical vapor deposition (CVD), reactive ion etching (RIE) and carbon calcination, to produce a palette of novel silicon-based material architectures. We discuss the design strategies and address the main features of the processing steps to provide a variety of new silicon-based materials with high morphological versatility and photonic quality. In particular, starting from three-dimensional (3D) hollow carbon opals, the porosity of the obtained architecture is easily controlled through the initial silicon infiltration, while open or closed surfaces are achieved mainly depending on the RIE conditions. The composition of the final structures is dependent on the sequence in which the processes are applied. As a result, pure Si and hybrid C-Si inverse structures with open or closed spheres at the top layer and with an optional passivation layer can be produced. Remarkably, choosing adequately the Si infiltration parameters and the calcination/RIE procedure, final structures with double-shell spheres are achieved.
\end{abstract}

\section{Introduction}

Hierarchically porous $3 \mathrm{D}$ materials with porosity in the micro, meso and macro ranges are receiving increasing attention from a wide range of research fields such as energy conversion and storage areas, ${ }^{1,2}$ catalysis, ${ }^{3,4}$ biomedicine,, 5 sensors, $, 7,8$ and optics,, 910 among others. ${ }^{11}$ The self-assembly of colloidal spheres is a straightforward approach to produce porous 3 D structures. ${ }^{12}$ In particular, hollow colloidal particles provide macroporosity (from their emptied core), and mesoporosity (from the interstices between them). Besides, the shell may allow creation of microporosity and surface functionalization, opening synthetic and functional diversity for such architectures. ${ }^{13,14}$ Hollow spheres have also been proposed in the last years in the photonic field for applications such as colloidal lithography, ${ }^{15}$ whispering gallery mode resonators ${ }^{16}$ and coating materials for tuning surface reflectivity. ${ }^{17}$ Research on colloidal spherical particles has mainly focused on silica, polystyrene or polymethylmethacrylate as building blocks, due to their straightforward fabrication, large availability and good control of their physicochemical properties. ${ }^{12}$ However, the increasing polydispersity of silica spherical particles larger than about $0.5 \mu \mathrm{m}$ and the lack of thermal, mechanical stability or solvent compatibility of polymeric particles have boosted the exploration of colloidal systems based on different materials. ${ }^{18,19}$ Additionally, the fabrication of new multifunctional, high-performance materials can allow a

a Instituto de Ciencia de Materiales de Madrid (ICMM); Consejo Superior de Investigaciones Científicas (CSIC)

b Inorganic Chemistry department, Chemical Sciences Faculty, Universidad Complutense de Madrid, 28040, Madrid, Spain.

c ICTS National Center for Electronic Microscopy, Universidad Complutense, 28040, Madrid, Spain.

${ }^{d}$ Department of Applied Physical Chemistry and Condensed Matter Physics Center (IFIMAC), Universidad Autónoma de Madrid 28049 Madrid, Spain

e IMDEA Nanoscience, Universidad Autónoma de Madrid, 28049 Madrid, Spain more versatile choice of the composition in order to circumvent the current limitations of the systems available to date. Specifically, Si and C are elements with the highest potential concerning cutting-edge technology. On the one hand, Si offers transparency in the near-infrared/infrared regions and high refractive index for light confinement and manipulation in optical sensing and optoelectronic circuits at telecommunication wavelengths. ${ }^{20,21}$ On the other hand, C provides high stability upon extreme processing conditions, and carbon spheres exhibit excellent monodispersity and size control..$^{22,23}$ It is therefore not only desirable but essential to develop synthetic strategies that integrate hollow particles based on these elements into materials with complex structures.

In this work, we demonstrate the fabrication of up to five types of hybrid C-Si and pure $\mathrm{Si}$ hierarchical structures starting from a $3 \mathrm{D}$ ordered template based on hollow carbon spheres, that is, a hollow carbon opal (HCO). ${ }^{22,24}$ Its selection as the starting material relies on its advantageous features to generate novel and improved architectures, conveniently combining properties in different areas of application such as:

i) Hierarchical porosity comprised of uniform, spherical macropores (the empty space inside the spheres), mesopores (the interstices between the spheres) and micropores (induced in the carbon shell by pyrolysis). Additionally, the $\mathrm{HCO}$ is a versatile system as it allows broad variation of fabrication parameters, such as the macropore size (from 500 to $900 \mathrm{~nm}$ ), the shell thickness (from 20 to $210 \mathrm{~nm}$ ) and the shell microporosity. ${ }^{22}$

ii) Photonic properties arising from the highly ordered fcc crystalline array formed by the spheres showing an intense Bragg peak (the lowest-energy photonic bandgap). ${ }^{24}$ This property not only allows applicability in photonics (as a sensitive platform for sensing for instance) but also constitutes a powerful diagnostic tool to follow the changes undergone by the structure along the fabrication process.

iii) Mechanical and thermal properties brought about by the closed-cell skeleton structure of the HCO in which air cavities are isolated, greatly improving robustness and mechanical stability. ${ }^{25}$ This characteristic, which contrasts with the interconnected air cavities in the traditionally reported inverse opals, is relevant to endow the template with excellent thermal and mechanical resilience that al- 


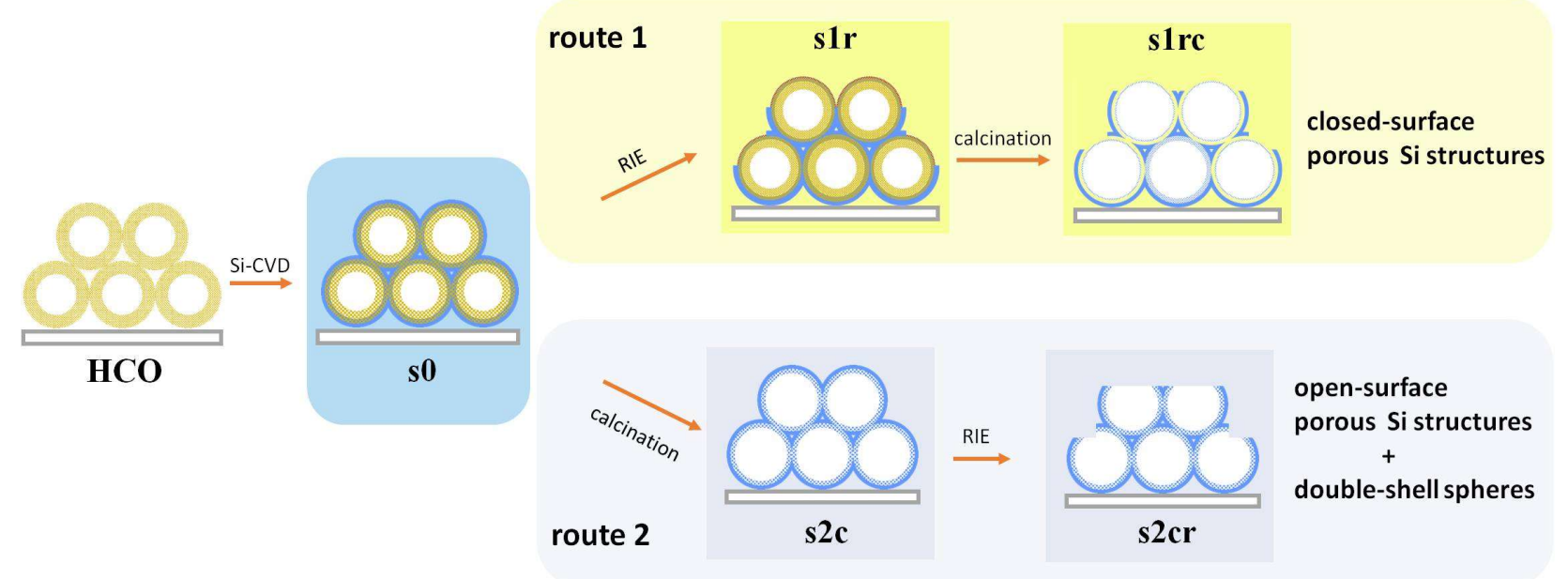

Scheme 1. Schematic fabrication process of C-Si and Si inverse opal structures based on a carbon photonic system. An HCO is Si-infiltrated to yield a hollow@CSi composite s0. RIE and subsequent calcination processes yield structures s1r and s1rc (route 1).Alternatively, calcination and subsequent RIE processes produce structures $\mathbf{s} \mathbf{2 c}$ and $\mathbf{s} \mathbf{2 c r}$, respectively (route 2). Yellow and blue colors represent $\mathrm{C}$ and $\mathrm{Si}$, respectively. Brown color in s1r denotes $\mathrm{CF}_{x}$ compounds (see text for details).

lows successive processing treatments ${ }^{26}$ without compromising the order, quality and stability of the resulting superstructures.

Hybrid C-Si and pure Si hierarchical superstructures were fabricated from HCO by performing a proper combination of three different processing steps: silicon growth by chemical vapor deposition (CVD), calcination, and reactive ion etching (RIE). Our results show the extraordinary variety in the morphology and photonic properties of the resulting structures by careful selection of the parameters defining each processing step. Indeed, the porosity of the final structure can be tuned through control of the Si CVD deposition inside the voids, while open or closed surfaces can be obtained by selecting the RIE conditions. Furthermore, and according the X-ray photoelectron spectroscopy (XPS) studies, the etching methodology may allow the concomitant fabrication of a passivating layer composed $\mathrm{SiO}_{x} \mathrm{~F}_{y}$, and a fluoropolymer $\left(\mathrm{CF}_{\mathrm{x}}\right)$, which can be very advantageous in sensing applications requiring selective reactivity. ${ }^{27,28}$

\section{Fabrication strategies}

Our starting system, the HCO, can be considered as formed by air spheres surrounded by porous carbon shells (hollow@carbon spheres); since these shells are in contact, the HCO constitutes a closed-cell structure. ${ }^{25,29}$ The proposed synthetic procedures involve up to three different steps, constituting two main routes (Scheme 1) -see Supporting Information for experimental details. In the initial step, common to both routes, $\mathrm{Si}$ is incorporated in and around the porous carbon shells by CVD, resulting in a hybrid opal formed by hollow spheres with a C-Si shell (as a result of the infiltration of $S i$ in the $C$ spheres) surrounded by a conformal pure Si layer (structure so). Following route 1 , so is subjected to an anisotropic RIE treatment, in order to controllably remove the conformal Si layer (structure s1r) and facilitate carbon removal, if desired, by subsequent calcination (structure sirc). Because of the presence of $\mathrm{C}$ during RIE, a passivating $\mathrm{CF}_{\mathrm{x}}$-rich surface is produced on the sir shells. This $\mathrm{CF}_{x}$ surface is resilient to and remains after the subsequent calcination, so that sirc consist of a porous silicon structure with closed surfaces. Alternatively, route 2 is accomplished by swapping these treatments to obtain different structures. Following this route, so is calcined in order to directly remove the carbon template, leaving a silicon porous shell and the surrounding Si layer. Thus, the inverse Si opal attained (structure s2c) is made of 'dual' walls: microporous -interior face of the shells- and dense external conformal layer- as will be further explained. A subsequent RIE treatment allows removal of the conformal layer and controllable opening of the microporous shell of the top layer, producing a porous silicon structure with open surfaces (structure s2cr). As a remarkable consequence of this route, and choosing adequately the Si infiltration parameters, final structures with double-shell spheres are achieved.

\subsection{Silicon infiltration}

The incorporation of $\mathrm{Si}$ in the HCO template proceeded via $\mathrm{Si}-\mathrm{CVD}$, following a standard methodology. ${ }^{30}$ The $\mathrm{Si}$ growth is essentially governed by two parameters, namely, the precursor gas $\left(\mathrm{S}_{2} \mathrm{H}_{6}\right)$ pressure and the temperature of decomposition. The gas pressure is varied between 110 and 530 Torr (see Section $S_{1}$ in Supporting Information for further details). The temperature affects both the Si growth rate and the formation of undesired particles..$^{31}$ As we are interested in controlling the amount of $\mathrm{Si}$ grown in the opals, low growth rates are required, ${ }^{32}$ so we selected a relatively low temperature of $365^{\circ} \mathrm{C}$. Figure 1 shows the $\mathrm{Si}$ infiltration of the HCO template to generate different so structures. The sphere arrangement is preserved, even after massive Si deposition, as confirmed by scanning electron microscopy (SEM) inspection (Figures 1a-d). The CVD working pressure allows easy control of the infiltration degree. The photonic response is also tuned and serves the additional purpose of monitoring the degree of $\mathrm{Si}$ infiltration of so by following its spectral changes (Figures 1e-h). The normal reflectance of the starting HCO template showed a well-defined Bragg peak, demonstrating the initial ordered arrangement. For any infiltration pressure, the 

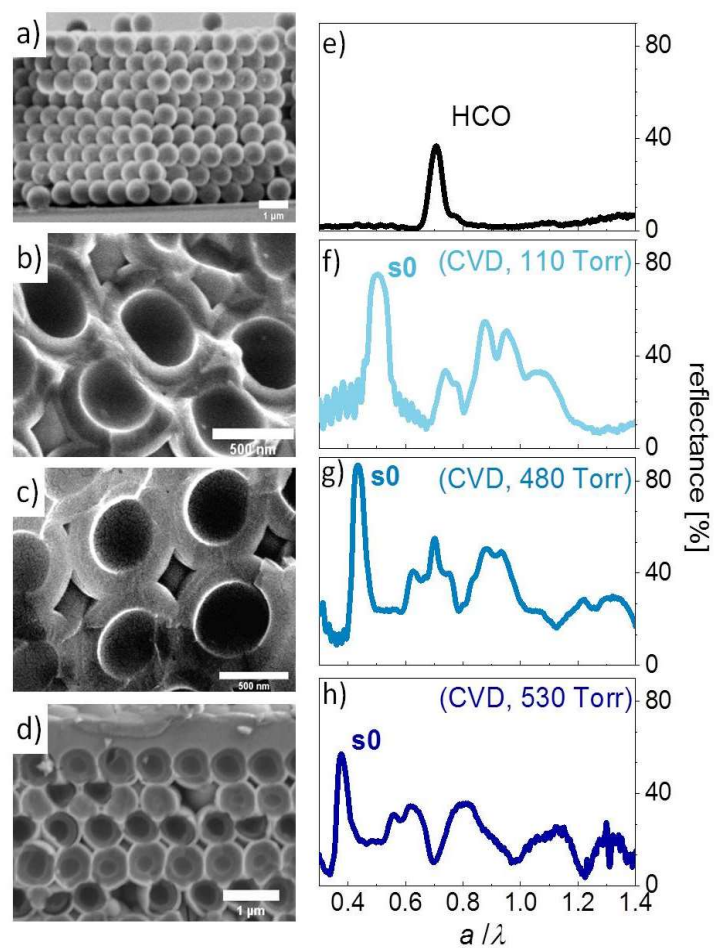

Figure 1. Si infiltration control by CVD. SEM images of (a) the starting HCO, (b-d) the resulting s0 structures after Si-infiltration at 110, 480 and 530 Torr. The images of $\mathbf{s} \mathbf{0}$ are cross-sections of the cleaved opal. (e-h) Corresponding optical reflectance. The spectra are taken at normal incidence and expressed versus energy in reduced units $(a / \lambda)$, where $a=\sqrt{2} D$ is the lattice constant ( $D$ is the sphere diameter) and $\lambda$ is the light wavelength.

Bragg peak shifted towards longer wavelengths (lower energies), as the average refractive index in the photonic structure increases due to the incorporation of silicon -the higher the infiltration pressure, the larger the redshift. Remarkably, and allowed by the increased dielectric contrast in the opal, an outstanding Bragg peak reflectance of $80 \%$ and more is achieved after infiltration at low (110 Torr) and medium (48o Torr) pressures (Figures if-g). At the highest pressure employed (530 Torr), a Si overlayer is formed on top of the structure (Figure $\mathrm{ih}$ ), capable of introducing new optical modes due to surface resonances..$^{33}$ Even more, at such pressure, irregular silicon deposits may be found on top of so, affecting the optical reflectance (e.g. reducing the Bragg peak intensity).

The progressive growth of $\mathrm{Si}$ in the multiporous $\mathrm{HCO}$ template is not trivial and can be described as follows. Disilane accessibility to the interior of the porous carbon shells is hampered by growth of the outer shell that tends to block the entrance to the pore network. Therefore, the CVD reaction in the $C$ porous shell stalls by exhaustion of disilane. Silicon can continue to grow freely on the outside of the spheres to build a conformal, dense layer enveloping them. The result of this process is sketched in Figure 2a. Only for long exposures ( $>1 \mathrm{~h}$, experiments not shown) or high pressures ( $>500$ Torr), the porous HCO network saturates and a thick, flat silicon overlayer is formed on the top of the sample.
Detailed information about the Si CVD growth process within the $C$ shell is revealed by high-angle annular darkfield imaging, scanning transmission electron microscopy (STEM-HAADF). Figure $2 \mathrm{~b}$ shows an HAADF image of a hollow@carbon system infiltrated with Si (at 110 Torr), in which several rings with different contrast can be observed. This contrast responds to both varying composition and varying density along with the thickness of each single material in different regions. The corresponding colored energy dispersive X-ray spectroscopy (EDS) mapping (Figure 2b) reveals the presence and location of $\mathrm{Si}, \mathrm{C}$ and $\mathrm{O}$ in the structure. Figure $2 \mathrm{c}$ shows a higher magnification HAADF image evidencing the different contrast areas along the marked line, from which the corresponding intensities of the EDS signals are depicted in Figure $2 \mathrm{~d}$ (the atomic ratios are listed in Table S1, Supporting Information). This analysis reveals an outermost layer of about $60 \mathrm{~nm}$ of the shell (zone 1, pink region in Figure 2d), formed mostly by Si. As the $\mathrm{O}-\mathrm{K}_{\alpha}$ map (in red) reveals, a minor amount of $\mathrm{O}$ is detectable at the outer shell surface, due to unavoidable oxidation. Beneath this Si-rich layer, a thin brighter interface of about $20 \mathrm{~nm}$ is detected in Figure 2c, (zone 2, blue region in Figure 2d), where $\mathrm{Si}$ and $\mathrm{O}$ are the main components, before reaching the $\mathrm{C}$ sphere zone. This region, highlighted
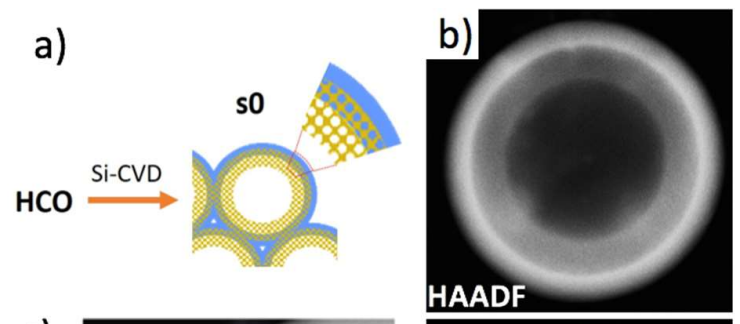

c)
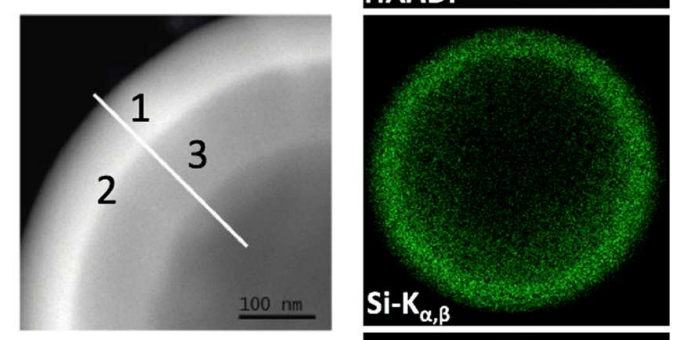

d)
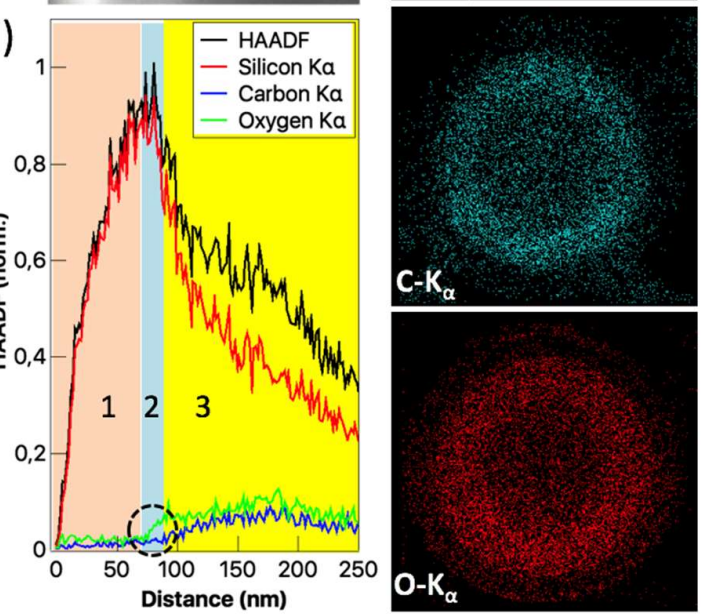

Figure 2. HAADF analysis of so. (a) Scheme of the Si infiltration process through the porous $C$ shells. (b) HAADF image and corresponding EDS maps of $\mathrm{Si}, \mathrm{C}$ and $\mathrm{O}$. (c) HAADF image at higher magnification. (d) Intensity variation of the EDS signals along the marked line in Figure $2 \mathrm{c}$. 

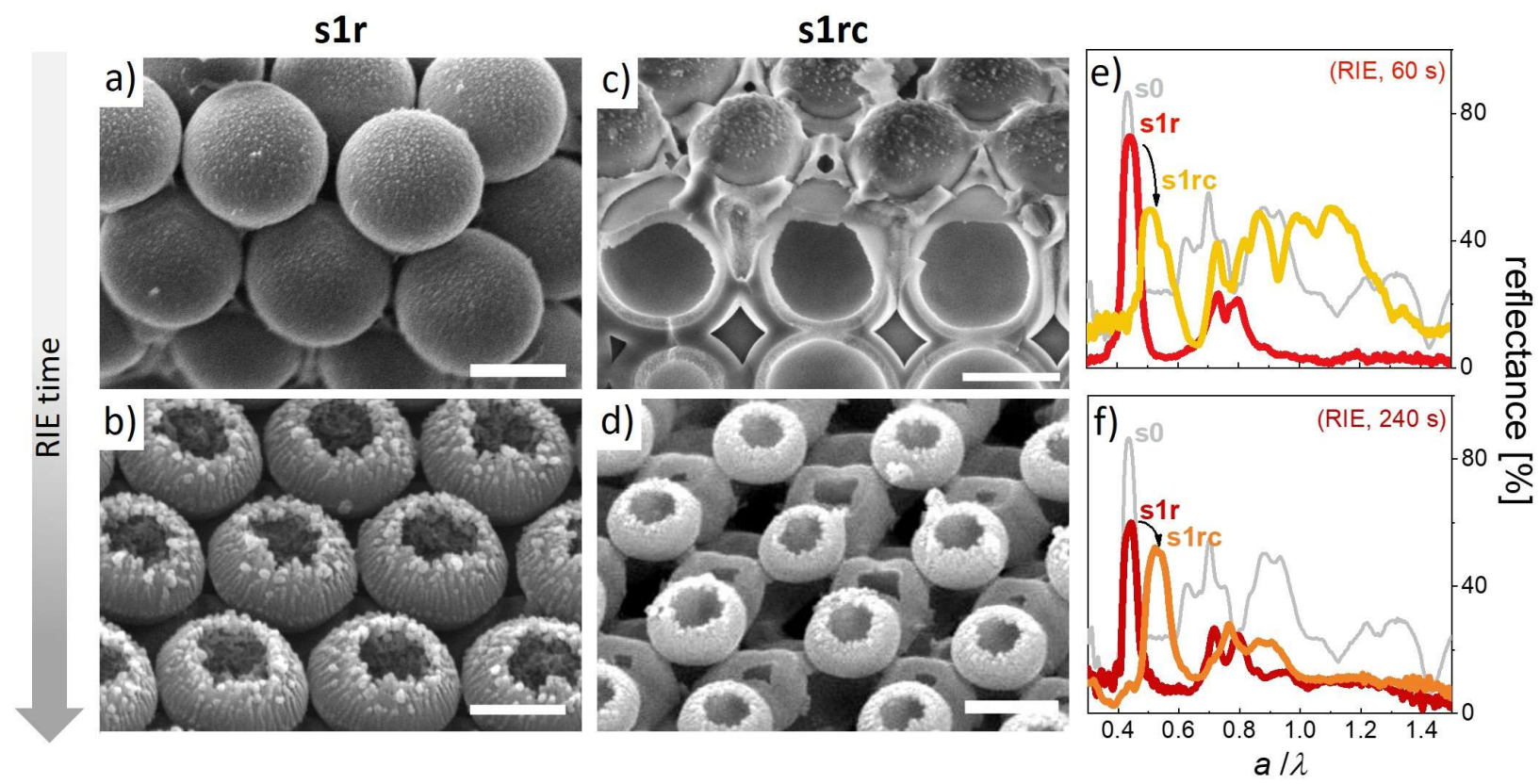

Figure 3. RIE etching and subsequent calcination (route 1) to produce s1r and s1rc structures with close or open surfaces. (a, b) SEM images of s1r structures after RIE time of a) $60 \mathrm{~s}$ and b) $240 \mathrm{~s}$. The initial s0 structure was Si-infiltrated at 110 Torr. (c,d) s1rc structures obtained after calcination of s1r structures previously infiltrated with Si at 480 Torr. SEM scale bars are $0.5 \mu \mathrm{m}$. (e, f) Compared optical reflectance, at normal incidence, of the structures obtained throughout the route 1.

with a dotted circle in Figure 2 d, plays a relevant role in the formation of double shell spheres, as will be further explained. The presence of this $\mathrm{Si}$ - and $\mathrm{O}$-rich area prior to the presence of $\mathrm{C}$ must be related to the CVD Si growing process on the roughed $\mathrm{C}$ surface, rich in O-containing functional groups susceptible to oxidize $\mathrm{Si}$ upon deposition, and forming $\mathrm{SiO}_{2}$. The presence of $\mathrm{C}$ is detected at around $80 \mathrm{~nm}$ deep from the outer Si surface, establishing the thickness of the grown Si layer. In this region (zone 3, yellow region in Figure 2d) C, O and Si coexist at different relative ratios from the outer to the inner part of the center of the sphere, according to previously mentioned Si infiltration mechanism sketched in Figure $2 a$.

In the following, we discuss two routes to generate different silicon structures with a tunable open surface. In particular, we focus on those systems obtained from so after Si infiltration at low and medium pressures.

\subsection{Route 1: Generation of closed-surface porous Si} structures

As sketched in Scheme 1, route 1 subjects the described structure so to anisotropic RIE for controlled removal of exposed areas of the deposited $\mathrm{Si}$, starting from the uppermost layers to achieve a hybrid HCO-Si opal with a tunable conformal layer on the spheres (structures sir). Both the $\mathrm{Si}$ infiltration degree and the RIE exposure time are essential fabrication parameters (Figure 3 and Figure S1 in Supporting Information) to tune the final structure. For example, RIE etching of a lightly infiltrated so sample (at 110 Torr) eliminates the conformal silicon layer after 6o s (structure sir, Figure za), while, for longer exposure times, the surface of the spheres on the top layer are eventually etched open (240 s, structure sir, Figure $3 \mathrm{~b}$ ). It must be noted that the $\mathrm{Si}$ overlayer present in heavily $\mathrm{Si}$-infiltrated samples can be removed using longer RIE exposure times (Supporting Information, Figure S2). Depending on the morphology resulting after RIE, the subsequent calcination yields sirc structures consisting of hollow@Si spheres with closed (Figure 3c) or open surface shells (Figure 3d). Interestingly, calcination leads to a systematic, significant collapse of the resulting hollow@Si spheres in the upper layer (see Figures $3 \mathrm{c}$ and $3 \mathrm{~d}$ ), while the bulk of the opal conserves the inverse structure (Figure $S_{3}$, Supporting Information).

Again, apart from many nuances and fine detail that requires numerical modelling to account for, the photonic response lets us follow the structural modifications occurred through the route 1 processing (Figures $3 e$ and $3 \mathrm{f}$ ). By comparing with so, the reflectance spectra of sir demonstrate a reduction in the Bragg peak intensity -the more, the longer the RIE exposure-, which is consistent with a decrease in the average refractive index as $\mathrm{Si}$ is gradually removed from the opal. Besides, the features at high energies are strongly reduced probably due to surface roughening. In the calcined sirc structures, the Bragg peak exhibits, along with reduced intensity, a clear blueshift (higher energy) due to the massive carbon elimination and the consequent decrease of the average refractive index of the porous shell, and widens due to the increase of the dielectric contrast.

The rather unexpected morphology of the top layer spheres in sir structures might be suspected to be due to material changes induced by the RIE process. In order to obtain a deeper insight, a careful XPS characterization was performed. Figure 4 depicts the complex line shape of $C_{1 s}$ spectrum obtained from structures sir and sirc, respectively. Peak assignment is based on binding energy values in comparison with those reported for a given chemical 

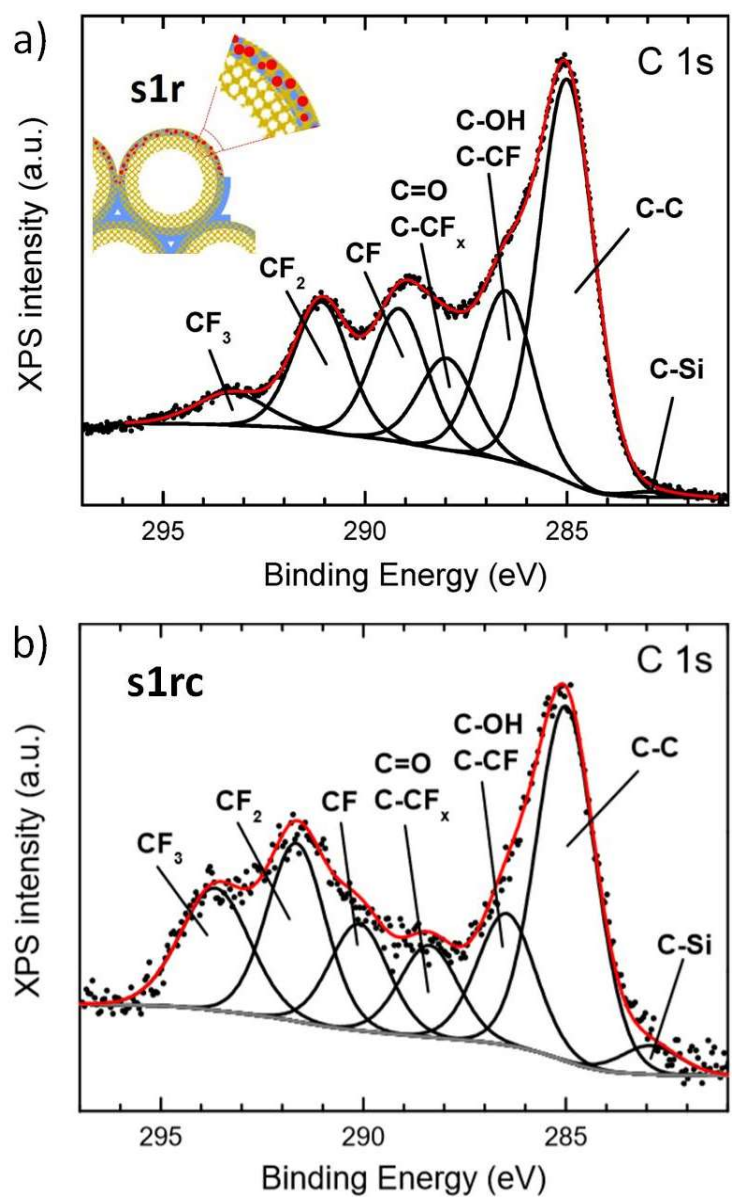

Figure 4. XPS characterization (route 1). Deconvolution of C1s XPS spectra of (a) s1r and (b) s1rc. Si-infiltration of s0 at 110 Torr, RIE exposure time was $60 \mathrm{~s}$. Inset in (a): sketch of the location of the species produced during RIE.

state. ${ }^{34}$ Full dots represent experimental data in the spectrum while solid lines represent Shirley background and component peaks. The fitting curve (red line) resulted from the sum of the several contributions belonging to: $\mathrm{C}-\mathrm{C}, \mathrm{C}-$ $\mathrm{H}, \mathrm{C}-\mathrm{OH}$ carbonyl groups, $\mathrm{O}-\mathrm{C}-\mathrm{O}$ carbonyl and $\mathrm{C}_{-} \mathrm{CF}_{x}, \mathrm{O}-$ $\mathrm{C}=\mathrm{O}$ carboxylic and $\mathrm{C}-\mathrm{F}, \mathrm{CF}_{2}$, and $\mathrm{CF}_{3}$ groups. Thus, since RIE involves the use of $\mathrm{SF}_{6}$ and $\mathrm{O}_{2}$ gasses, a new set of $\mathrm{C}-\mathrm{F}$ chemical species are formed during the etching (sketch in Figure 4a); note, however, that the largest contribution is represented by the $\mathrm{C}-\mathrm{C}$ species. In addition, the analysis of Fis spectrum reveals the existence of two components, a main one being ascribed to $\mathrm{CF}_{x}$ groups in agreement to previous $C_{1 s}$ results and a small contribution coming from $\mathrm{Si}-\mathrm{F}$ chemical environment. The binding energy values of these signals are in accordance with values reported. ${ }^{34}$ On the other hand, the deconvolution of Sizp core level also confirms the majority presence of $\mathrm{SiO}_{x} \mathrm{~F}_{y}$ compounds together with weak signals of $\mathrm{SiO}_{x}$ and $\mathrm{SiC}$. Both the intensity and binding energy shift of the main component with respect to the $\mathrm{SiO}_{x}$ emission ${ }^{34}$ indicate that the outermost passivation layer is mostly composed of $\mathrm{SiO}_{x} \mathrm{~F}_{y}$ (Figure $\mathrm{S}_{4}$, Supporting Information).

It is then clear that the fluorinated precursor $\mathrm{SF}_{6} / \mathrm{O}_{2}$ reacts with both $\mathrm{C}$ and $\mathrm{Si}$ during $\mathrm{RIE}$ and builds a $\mathrm{CF}_{x} / \mathrm{SiO}_{x} \mathrm{~F}_{y}$ passivation layer. Interestingly, Figure $4 \mathrm{~b}$ evidences that
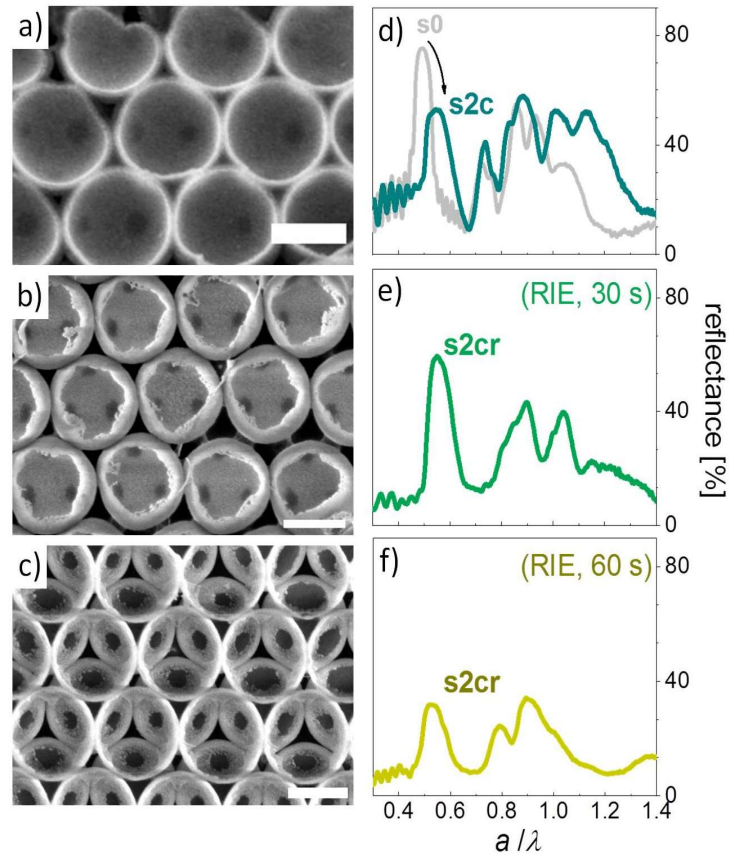

Figure 5. Carbon calcination and subsequent RIE etching (route 2). (a) SEM images of (a) s2c and (b, c) s2cr samples after RIE treatment during 30 and $60 \mathrm{~s}$, respectively. SEM scale bars are $0.5 \mu \mathrm{m}$. (d-f) Compared optical reflectance at normal incidence of the structures obtained throughout the route 2. The initial so structure was Si-infiltrated at 110 Torr.

the $\mathrm{CF}_{x}$ species are still present in the calcined sirc structure and, additionally, that a new small contribution related to $\mathrm{C}-\mathrm{Si}$ is formed. The persistence of $\mathrm{CF}_{x}$ in sirc is not surprising, since it is known that fluoropolymers are extremely inert and resistant. The relative contents of $\mathrm{Si}, \mathrm{C}$, $\mathrm{O}$, and $\mathrm{F}$ (table $\mathrm{S} 2$, Supporting Information) show that the main loss upon calcination is related to $C$ and $F$ (free $F$, not forming part of the $\mathrm{CF}_{x}$ compounds that, although in a different proportion, are still present in the final structure). Thus, the observed sphere shrinkage, which is more obvious in the top layer, may be related to the decreased content of $\mathrm{C}$ and $\mathrm{F}$ in the final sirc structure.

\subsection{Route 2: Generation of open-surface porous Si} structures

Alternatively to route 1 , a route 2 can be chosen (see Scheme 1), in which the so structure is first calcined to remove $C$ and obtain a purely $\mathrm{Si}$ inverse opal (structure s2c). SEM inspection of s2c (Figure 5a) already suggests the elimination of $C$ shell upon calcination. In fact, the image shows small windows that connect adjacent spheres upon calcination, indicating an interconnected network of air spheres surrounded by silicon shells, that is, a well-known silicon inverse opal. Carbon removal is confirmed, on the one hand, by HRTEM, including HAADF images and EDS mapping of a sic structure (Figure $\mathrm{S}_{5}$, Supporting Information), and, on the other hand, by XPS (less than $4 \%$ of C remaining). The structure may be further tailored by the RIE process to open the Si surfaces (s2cr structures). The final morphology of these open-shell Si structures depends on both the $\mathrm{Si}$ infiltration degree and the extent of the etching procedure (Figures $5 \mathrm{~b}-\mathrm{c}$ after 30 and $60 \mathrm{~s}$ RIE, respec- 

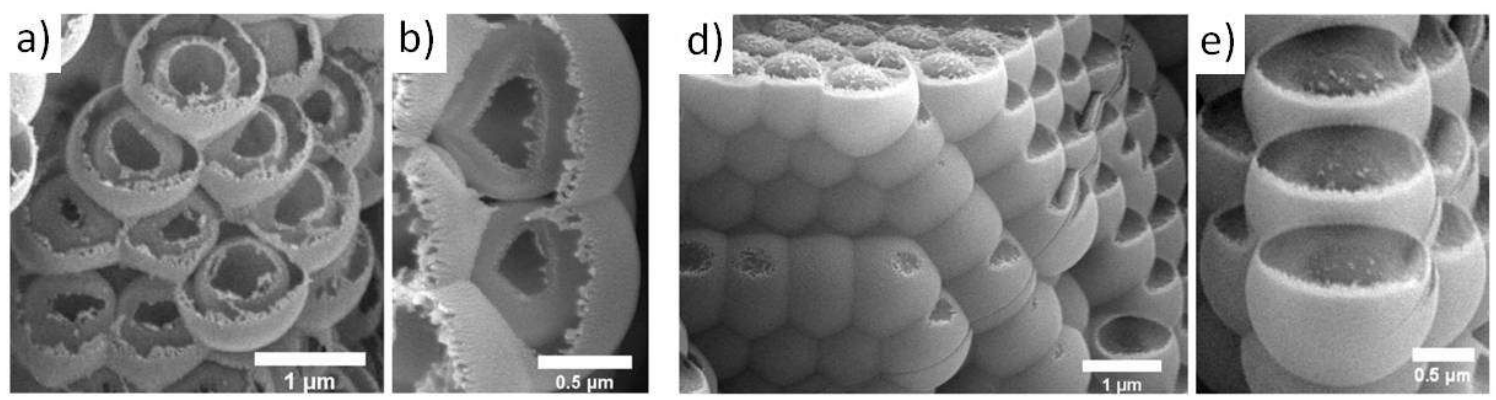

c)
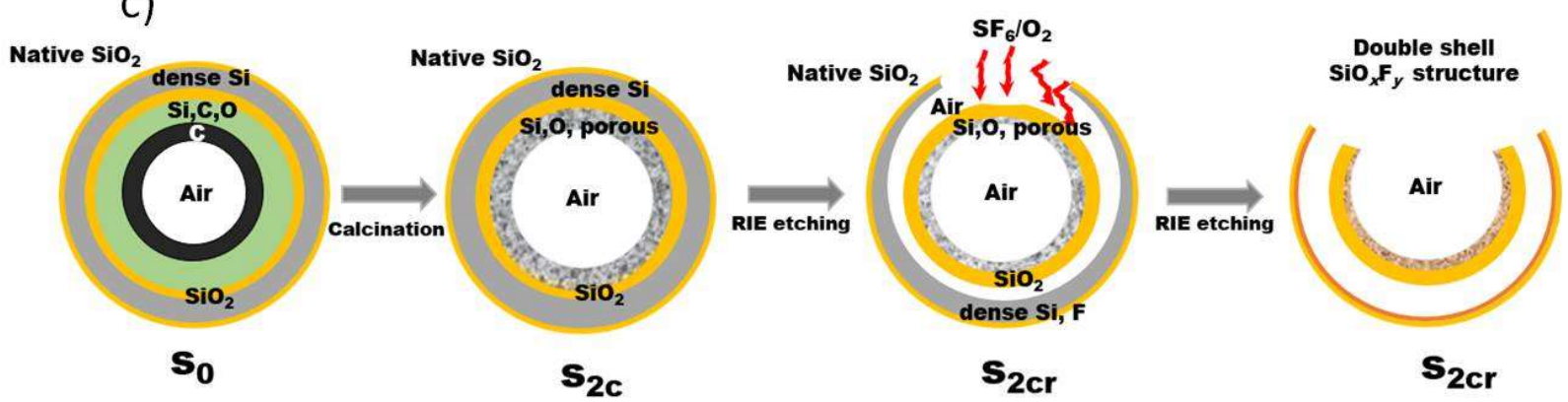

Figure 6. Generation of double Si shells. (a,b) SEM images at different magnifications of s2cr samples (c) Sketch of the proposed mechanism explaining the generation of double-shell Si spheres upon RIE etching. (d, e) SEM images at different magnifications of s2cr samples evidencing the different etching rate of the RIE process across the structure.

tively). In structures with a low Si infiltration, the RIE process eventually leads to a grating-like geometry, in which the structure, thinner regions wiped out by the effect of the etching, clearly exhibits well-exposed windows interconnecting the silicon shells interiors (Figure $5 \mathrm{c}$ ). The opening of these windows (hence their diameter) is ultimately determined by the RIE exposure time.

Once again, a good photonic response allows tracing the structural modifications occurred through route 2 (Figures 5d-f). Compared to so, the Bragg peak of s2c shows a blueshift (larger energies) and a reflectance decrease, in agreement with the decreased average refractive index in the structure upon carbon removal. After RIE, the position of the Bragg peak in s2cr remains unaffected, while the features in the high-energy range decrease (as observed in Figure 3) probably with the surface modifications.

Interestingly, the resulting s2cr structures may exhibit double Si shells (Figure 6a and 6b). This double shell is associated with the etching process across the structure containing different layer thicknesses, compositions, and density. The suggested etching mechanism is depicted in Figure $6 c$, that summarizes the whole progress from so to s2r structures. In this process, anisotropic RIE etching from the top layer attacks through the thin native $\mathrm{SiO}_{2}$ passivating layer, and gradually eliminates the dense $\mathrm{Si}$ shell from the top downwards. This etching proceeds at a different rate than that across the $20 \mathrm{~nm}$ interface $\mathrm{SiO}_{2}$ layer previously explained in Figure 2. This effect is well observed in Figures $6 c$ and $6 \mathrm{~d}$, showing evidences of the removal of dense $\mathrm{Si}$ prior to the removal of the part near the center of the spheres (the $\mathrm{SiO}_{2}$ interface). Notice, besides, that those rates may also vary during etching, since, as previously mentioned and according to XPS, (Figure $\mathrm{S}_{4}$, Supporting
Information) new $\mathrm{SiO}_{x} \mathrm{~F}_{y}$ and F-Si species can be dynamically formed.

\section{Conclusions}

In this work we present fabrication routes that, applied to $\mathrm{Si}$ infiltrated hierarchically porous $\mathrm{C}$ self-assembled colloidal crystals (hollow@C opal), lead to several silicon-based photonic architectures. These processes rely on the combination of Si chemical vapor deposition, reactive ion etching and calcination steps. The sequence of processes performed and their governing parameters were decisive for the morphological and optical properties of the final structures. Therefore, the selection of the fabrication route and control of the applied parameters directly provide a rich palette of superstructures with easy tunability. Thus, the calcination and subsequent RIE of a previously Si infiltrated sample yields a solid silicon inverse structure that could be finely tailored through the whole thickness, with a double $\mathrm{Si}$ shell. The generation of original double-shell Si inverse opals was demonstrated by choosing specific fabrication conditions and process sequence, which involves a complex interplay between RIE and the structure, for which a mechanism is proposed. Alternatively, the application of RIE prior to the calcination led to inverse structures with a closed top layer of shrunk spheres, whose composition included fluoropolymers. Importantly, such shrinkage did not compromise the opal stability, keeping the order and its optical response in the bulk structure. This study highlights the wide range of possibilities offered by general selfassembly-templating techniques in materials science.

\section{Acknowledgements}

This work was partially funded by the Spanish MCIU CSIC13-4E-1794, MAT2014-58731-JIN, MAT2016-80394-R, MAT2017-85617-R and RTI2018- 
093921-B-C41 and the excellence programs MDM-2014-0377 and SEV2016-0686. The authors acknowledge the service from the MiNa Laboratory at IMN, and funding from Comunidad de Madrid project S2018/NMT4291 (TEC2SPACE). We thank the National Center for Electron Microscopy

1 Z. Li, K. Zhang, M.-Y. Li, C.-L. Liu, W.-S. Dong, J. Porous Mater. 2018, 25, 1047.

2 S. Rehman, S. Guo, Y. Hou, Adv. Mater. 2016, 28, 3167.

3 G. Collins, E. Armstrong, D. McNulty, S. O'Hanlon, H. Geaney, C. O’Dwyer, Sci. Technol. Adv. Mater. 2016, 17, 563.

4 L. Yu, H. Bin Wu, X. W. D. Lou, Acc. Chem. Res. 2017, 50, 293.

5 S. Jung, J. L. Kaar, M. P. Stoykovich, Mol. Syst. Des. Eng. 2016, 1, 225.

6 Y. Si, M. Chen, L. Wu, Chem. Soc. Rev. 2016, 45, 690.

7 J. Kuang, L. Liu, Y. Gao, D. Zhou, Z. Chen, B. Han, Z. Zhang, Nanoscale 2013, 5, 12171.

8 F. Gallego-Gómez, M. Morales, M., A. Blanco, C. López, Adv. Mater. Technol. 2019, 1800493.

9 F. Gallego-Gómez, M. Ibisate, D. Golmayo, F. J. Palomares, M. Herrera, J. Hernández, S. I. Molina, A. Blanco, C. López, Adv. Mater. 2011, 23, 5219

10 K. Kitano, K. Suzuki, K. Ishizaki, S. Noda, Phys. Rev. B - Condens. Matter Mater. Phys. 2015, 91, 1.

11 X.-Y. Yang, L.-H. Chen, Y. Li, J. C. Rooke, C. Sanchez, B.-L. Su, Chem Soc. Rev. 2017, 46, 481.

12 J. F. Galisteo-López, M. Ibisate, R. Sapienza, L. S. Froufe-Pérez, Á. Blanco, C. López, Adv. Mater. 2011, 23, 30.

13 S. Li, A. Pasc, V. Fierro, A. Celzard, J. Mater. Chem. A 2016, 4, 12686.

14 C. Dai, A. Zhang, J. Li, K. Hou, M. Liu, C. Song, X. Guo, Chem. Commun. 2014, 50, 4846.

15 K. Zhong, J. Li, S. Van Cleuvenbergen, K. Clays, Nanoscale 2016, 8, 15845

16 S. Yang, Y. Wang, H. D. Sun, Adv. Opt. Mater. 2015, 3, 1136.

17 Z. Xing, S. W. Tay, Y. H. Ng, L. Hong, ACS Appl. Mater. Interfaces 2017, 9, 15103.

18 Z. Xue, P. Wang, A. Peng, T. Wang, Adv. Mater. 2018, 1801441, 1801441.

19 S. Li, A. Pasc, V. Fierro, A. Celzard, J. Mater. Chem. A 2016, 4, 12686.
(CNME; UCM, Madrid) for facilities. L.K.G. acknowledges COLCIENCIAS doctoral grant. A. Rodríguez Barbero of ICV is kindly acknowledged for support in SEM characterization.

\section{References}

20 G. Roelkens, et al., Photonics 2015, 2, 969.

21 A. Rahim, T. Spuesens, R. Baets, W. Bogaerts, Proc. IEEE 2018, 106, 2313.

22 L. K. Gil-Herrera, Á. Blanco, B. H. Juárez, C. López, Small 2016, 12, 4357.

23 M. D. Goodman, K. A. Arpin, A. Mihi, N. Tatsuda, K. Yano, P. V. Braun, Adv. Opt. Mater. 2013, 1, 300.

24 L K. Gil-Herrera, J. A. Pariente, F. Gallego-Gómez, F. Gándara, B. H. Juárez, Á. Blanco, C. López, Adv. Funct. Mater. 2018, 28, 1703885

25 X. Chen, L. Wang, Y. Wen, Y. Zhang, J. Wang, Y. Song, L. Jiang, D. Zhu, J. Mater. Chem. 2008, 18, 2262.

26 Y. Zhang, J. Wang, Y. Huang, Y. Song, L. Jiang, J. Mater. Chem. 2011, 21, 14113.

27 G. T. Dobbs, B. Balu, C. Young, C. Kranz, D. W. Hess, B. Mizaikoff, Anal. Chem. 2007, 79, 9566

28 V. Singh, P. T. Lin, N. Patel, H. Lin, L. Li, Y. Zou, F. Deng, C. Ni, J. Hu, J. Giammarco, A. P. Soliani, B. Zdyrko, I. Luzinov, S. Novak, J. Novak, P. Wachtel, S. Danto, J. D. Musgraves, K. Richardson, L. C. Kimerling, A. M. Agarwal, Sci. Technol. Adv. Mater. 2014, 15, 014603.

29 G. Guan, K. Kusakabe, H. Ozono, M. Taneda, M. Uehara, H. Maeda, J. Mater. Sci. 2007, 42, 10196.

30 Á. Blanco, E. Chomski, S. Grabtchak, M. Ibisate, S. John, S. W. Leonard, C. Lopez, F. Meseguer, H. Miguez, J. P. Mondia, G. A. Ozin, O. Toader, H. M. van Driel, Nature 2000, 405, 437.

31 R. Fenollosa, M. Garín, F. Meseguer, Phys. Rev. B 2016, 93, 235307.

32 F. García-Santamaría, M. Ibisate, I. Rodríguez, F. Meseguer, C. López, Adv. Mater. 2003, 15, 788

33 F. García-Santamaría, E. C. Nelson, P. V. Braun, Phys. Rev. B 2007, 76, 0751321.

34 J. Pereira, L. E. Pichon, R. Dussart, C. Cardinaud, C. Y. Duluard, E. H. Oubensaid, P. Lefaucheux, M. Boufnichel, P. Ranson, Appl. Phys. Lett. 2009, 94, 3. 